\title{
Causes and Predictors of Early Hospital Readmission in Systemic Lupus Erythematosus
}

\author{
Angelica Nangit, Connie Lin, Mariko L. Ishimori, Brennan M.R. Spiegel, \\ and Michael H. Weisman
}

ABSTRACT. Objective. We investigated characteristics of adult patients with systemic lupus erythematosus (SLE) readmitted to the hospital within 30 days of discharge, in an attempt to identify the causes of early readmission.

Methods. We performed a retrospective case-control study examining all inpatient electronic health records of patients with SLE at Cedars-Sinai Medical Center over a 2.5-year period (2012-2014). Patients were included if they had an International Classification of Diseases, 9th ed diagnosis of SLE and were readmitted within 30 days of their initial hospitalization. Patients with SLE not readmitted during this time period were used as a control group. Demographic and clinical variables for each patient were collected, and we used the Charlson Comorbidity Index to characterize comorbidities. The Systemic Lupus International Collaborating Clinics/American College of Rheumatology Damage Index (SDI) was used to assess the chronic damage of SLE. Stepwise multivariable logistic regression analysis was used to predict factors associated with readmission.

Results. In total, 570 hospitalizations representing 455 unique patients met our inclusion and exclusion criteria. Of these, 154 patients (34\%) underwent readmission within 30 days of their initial hospitalization. Patients in the early readmission group were more likely to have government-sponsored Medicaid insurance and were significantly associated with an increased SDI (OR 1.27, 95\% CI 1.1-1.48), lower serum hemoglobin (OR 0.82, 95\% CI 0.72-0.93), and lower serum albumin (OR $0.66,95 \%$ CI $0.47-0.91)$.

Conclusion. One-third of hospitalized patients with SLE were readmitted within 30 days at our institution. We identified characteristics of this at-risk population at time of discharge with high specificity, in hopes of reducing this costly outcome. (First Release April 15 2018; J Rheumatol 2018;45:929-33; doi:10.3899/jrheum.170176)

Key Indexing Terms:

SYSTEMIC LUPUS ERYTHEMATOSUS

Systemic lupus erythematosus (SLE) is a chronic immune-mediated disease predominantly affecting women, displaying highly variable disease phenotypes and comorbidities and often requiring inpatient hospitalization. Each year, about $20-25 \%$ of individuals with SLE are hospitalized ${ }^{1}$. Most of these hospitalizations are observed to be related to either clinical flare of SLE, infection, thromboembolic disease, or associated medical comorbidities ${ }^{2,3}$. Notably, SLE has the sixth highest 30-day readmission rate among all medical conditions in the United States ${ }^{2}$, and early hospital readmissions have been recognized as a common and costly occurrence.

From the Departments of Rheumatology, Internal Medicine, and Gastroenterology, Cedars-Sinai Medical Center, Los Angeles, California, USA.

A. Nangit, MD, Rheumatology, Cedars-Sinai Medical Center; C. Lin, MD, Internal Medicine, Cedars-Sinai Medical Center; M.L. Ishimori, MD, Rheumatology, Cedars-Sinai Medical Center; B.M. Spiegel, MD,

Gastroenterology, Cedars-Sinai Medical Center; M.H. Weisman, MD, Rheumatology, Cedars-Sinai Medical Center.

Address correspondence to Dr. M.H. Weisman, Cedars-Sinai Medical Center, 8700 Beverly Blvd., Room B-122, Los Angeles, California 90048, USA.E-mail:weisman@cshs.org

Accepted for publication January 16, 2018.

\section{HOSPITAL READMISSION READMISSION}

Research demonstrates a significant economic burden related to SLE patient care, largely due to high healthcare use $^{4}$. Carefully done studies have investigated patient demographics that were associated with issues of primary hospitalization and mortality in patients with $\operatorname{SLE}^{5,6,7,8}$; however, few address the causes of hospital readmission in this population. Analyses of early readmissions highlight significant quality-of-care problems in patients with chronic diseases such as SLE. Obtaining an adequate grasp of the characteristics of early readmission for patients with SLE may allow for effective planning of transitions in care, as well as for resource use. Working in a high-volume SLE clinical care and research facility in Los Angeles with a mature electronic medical health record system, we are positioned to investigate the clinical characteristics of adult patients with SLE who are readmitted to Cedars-Sinai Medical Center (CSMC) within 30 days of prior discharge to identify potentially preventable readmissions. These early readmissions highlighted deficient areas in quality-related care, as well as identified characteristics and processes associated with readmission.

Personal non-commercial use only. The Journal of Rheumatology Copyright $\odot$ 2018. All rights reserved. 


\section{MATERIALS AND METHODS}

Study settings and patients. We performed a retrospective case-control study examining all inpatient electronic health records of patients with SLE at CSMC over a defined period of time. We queried the CSMC database for patients with an International Classification of Diseases, 9th ed (ICD-9) diagnosis of SLE (710.0) as either a primary or secondary diagnosis. Additionally, charts were reviewed to confirm whether patients met the American College of Rheumatology SLE classification criteria; however, because of missing data, given that this is a cross-sectional study and some patients were seeing rheumatologists outside of CSMC, we were unable to confirm whether all these patients truly met criteria. Patients were included into our study case group if they met the following criteria: (1) an ICD-9 code of 710.0; (2) were readmitted within 30 days of their index hospitalization; and (3) were admitted between January 1, 2012, and July 31, 2014. These readmitted patients were compared to control patients with SLE who did not undergo readmission during the same time period. Early readmission was defined as readmission within 30 days after the discharge date of the patient's index hospitalization date in this time period. Patients who were not hospitalized, were discharged from the emergency room, or died during hospitalization were excluded. Additionally, all admissions were characterized as planned or unplanned. A hospitalization was identified as planned if there is mention in the patient's record of an intentional hospitalization for purposes of a procedure or inpatient rehabilitation. A hospitalization was deemed as unplanned if a patient was admitted for acute medical care because of a clinical condition ${ }^{9}$. Approval for this study was not required in accordance with the policy of CSMC.

Predictor variables. Variables of interest included age, sex, ethnicity, insurance payer, types of clinical problems addressed during index hospitalization, and laboratory values. If multiple laboratory values were collected during the index hospital stay, the laboratory value that was collected closest to the discharge date of the index admission was recorded. Use of steroids in the hospital was recorded. If steroids were administered inpatient, then the average daily dose throughout the hospital stay was calculated. If the patient was discharged from the index admission while taking steroids, the discharge dose was also noted during data collection. Additionally, it was noted whether patients were taking immunosuppressive medications prior to the index hospitalization. We calculated the Charlson Comorbidity Index to characterize comorbidity disease status in each patient ${ }^{10}$, using the diagnoses listed in the patient's chart. To assess the chronic damage of SLE, we used the Systemic Lupus International Collaborating Clinics/American College of Rheumatology Damage Index (SDI), which measures damage after the diagnosis of SLE ${ }^{11}$. The SDI assesses 12 systems; damage is defined as nonreversible change that has occurred since the onset of SLE, and historically higher damage index scores have been associated with a poor prognosis. Because (1) there is a wide range of factors that predict outcomes and affect mortality in patients with SLE, and (2) SLE disease phenotypes display remarkable heterogeneity, we developed an a priori conceptual model to identify the variables likely to be associated with readmission (Appendix 1). We then recorded these prespecified patient characteristics, clinical data, procedural data, and medical therapies used during each hospitalization through review of physician progress notes, nursing records, and procedure reports, as well as laboratory and imaging reports.

Analyses. Patients with SLE were stratified into 2 groups: those readmitted within 30 days of the initial discharge following index hospitalization versus those not readmitted. All predictor variables were compared between these 2 groups. For baseline characteristics, $\mathrm{p}$ values were calculated using a 2-tailed $\mathrm{T}$ test for continuous data and a chi-squared test for categorical data. $\mathrm{P}$ values were calculated using a 2 -tailed $\mathrm{T}$ test, and a $\mathrm{p}$ value $<0.05$ indicated significance. Stepwise multivariable logistic regression analysis was used to develop a parsimonious model that maximized pseudo $\mathrm{R}^{2}$ in predicting 30-day readmission. We calculated the OR and 95\% CI for final predictors in the model. Receiver-operating characteristic (ROC) curve analysis identified a cutoff probability that maximized predictive discrimination between readmission versus nonreadmission. We report the sensi- tivity, specificity, positive predictive value (PPV), and negative predictive value (NPV) of the model in distinguishing between groups, along with the $\mathrm{C}$ statistic for the area under the ROC curve.

\section{RESULTS}

In total, 570 hospitalizations that met our inclusion and exclusion criteria were reviewed. Because of the presence of repeat readmissions in the same patient, our final dataset consisted of 455 unique patient records. In the study group, 154 patients (34\%) underwent early readmission, while 301 patients did not undergo any readmissions during the predefined study period; the latter set of patients was used as the control/comparison group. Table 1 provides the baseline characteristics of the full cohort. The mean age of the patients was similar for the readmission group and control group (52.6 yrs and $51.2 \mathrm{yrs}$, respectively). The majority of patients were female in both groups ( $89.6 \%$ readmission vs $90.7 \%$ control). The early readmission group, as compared to the control group, contained a higher percentage of African Americans and Asians; whites, however, were significantly more abundant in the nonreadmission group. When insurance was considered, the early readmission group had more Medicaid (government medical insurance) and Medicare (medical insurance for the elderly) patients compared to the control group. In particular, the early readmission group had significantly more patients on Medicaid ( $p=0.0075$ ) or the combination of Medicare and Medicaid $(\mathrm{p}=0.0008)$.

In the early readmission group, the vast majority of patients $(69 \% ; n=106)$ were readmitted only once. However, 21 patients were readmitted twice, 12 patients were readmitted 3 times, 7 patients were readmitted 4 times, and 8 patients were readmitted $\geq 5$ times during the course of the 2.5 -year study period. The maximum number of readmissions was 12 times, which was seen in only 1 patient. Only a small minority of readmissions $(15.6 \%)$ was classified as planned readmission. The discharge inpatient steroid dose tended to be higher in the early readmission group.

Factors that affected hospital readmission are shown in Table 2. Additionally, the causes and indications for index admission and readmission were collected; however, no statistical significance was seen between the 2 groups. Patients in the early readmission group were more likely to have Medicaid insurance (OR 1.93, 95\% CI 0.98-3.78), and were significantly associated with an increased SDI (OR $1.27,95 \%$ CI $1.1-1.48, \mathrm{p}=0.001)$, lower serum hemoglobin (OR 0.82, 95\% CI 0.72-0.93, $\mathrm{p}=0.002$ ), and lower serum albumin (OR $0.66,95 \%$ CI $0.47-0.91, \mathrm{p}=0.012$ ). The final stepwise regression model explained $16 \%$ of the variance in readmission. ROC analysis yielded an area under the curve of 0.76 . Using a probability threshold of 0.9 on the logit model, the sensitivity, specificity, PPV, and NPV for 30 -day readmission were $9 \%, 99 \%, 92 \%$, and $45 \%$, respectively. Thus, the score was highly specific at this threshold, but not sensitive; it is a tradeoff that allows focused attention on those with a high score (and thus very likely to

Personal non-commercial use only. The Journal of Rheumatology Copyright $\odot$ (2018. All rights reserved 
Table 1. Baseline characteristics of patients readmitted within 30 days of index admission versus nonreadmitted patients. Values are mean or $\mathrm{n}(\%)$ unless otherwise specified.

\begin{tabular}{|c|c|c|c|}
\hline Characteristics & Early Readmission, $\mathrm{n}=154$ & Not Readmitted, $\mathrm{n}=301$ & $\mathrm{p}$ \\
\hline Age, yrs & 52.6 & 51.2 & 0.4 \\
\hline Female & $138(89.6)$ & $273(90.7)$ & 0.7 \\
\hline \multicolumn{4}{|l|}{ Ethnicity } \\
\hline White & $66(42.9)$ & $157(52.2)$ & 0.0001 \\
\hline Hispanic & $28(18.2)$ & $64(21.3)$ & 0.33 \\
\hline African American & $42(27.3)$ & $56(18.6)$ & 0.07 \\
\hline Asian & $16(10.4)$ & $18(6.0)$ & NS \\
\hline Unknown & $2(1.3)$ & $5(1.7)$ & NS \\
\hline \multicolumn{4}{|l|}{ Insurance } \\
\hline Medicare* & $9(5.8)$ & $15(5.0)$ & 0.31 \\
\hline Medicare + private & $32(20.1)$ & $62(20.6)$ & 0.9 \\
\hline Medicaid** & $17(11.0)$ & $21(7.0)$ & 0.0075 \\
\hline Medicare-Medicaid & $35(22.7)$ & $51(16.9)$ & 0.0008 \\
\hline Private & $54(35.7)$ & $142(47)$ & 0.0004 \\
\hline Uninsured & $7(4.5)$ & $10(303)$ & 0.71 \\
\hline \multicolumn{4}{|l|}{ Biochemical $^{1}$} \\
\hline Albumin, g/dl & 3.47 & 3.81 & $<0.0001$ \\
\hline Creatinine, $\mathrm{mg} / \mathrm{dl}$ & 2.3 & 1.15 & $<0.0001$ \\
\hline Hemoglobin, g/dl & 10.1 & 11.2 & $<0.0001$ \\
\hline Immunosuppressive use & $102(66.2)$ & $150(50)$ & 0.001 \\
\hline Steroid dose inpatient ${ }^{2}$ & 32.2 & 28.4 & 0.27 \\
\hline Steroid dose outpatient ${ }^{3}$ & 9.5 & 6.9 & 0.24 \\
\hline $\mathrm{CCI}$ & 4.79 & 3.31 & NS \\
\hline SDI & 2.75 & 1.58 & NS \\
\hline
\end{tabular}

* Government medical insurance for the elderly. ** Government medical insurance. ${ }^{1}$ All laboratory values were included in average calculation and may include multiple values for the same patient. ${ }^{2}$ Steroid dose inpatient was calculated by summing the total $\mathrm{mg}$ of prednisone (or prednisone equivalent) during hospital course and dividing it by the length of stay. ${ }^{3}$ Steroid dose outpatient was the $\mathrm{mg}$ of prednisone (or prednisone equivalent) patient was taking at discharge. NS: not significant; CCI: Charlson Comorbidity Index; SDI: Systemic Lupus International Collaborating Clinics/American College of Rheumatology Damage Index.

Table 2. OR of 30-day readmission in patients with SLE at CSMC.

\begin{tabular}{lcc}
\hline Variables & OR $(95 \% \mathrm{CI})$ & $\mathrm{p}$ \\
\hline Medicaid insurance* & $1.93(0.98-3.78)$ & 0.057 \\
Clinical characteristics & & \\
$\quad$ No prior immunosuppressives & $0.61(0.40-0.94)$ & 0.026 \\
$\quad$ Unplanned index admission & $1.54(0.94-2.49)$ & 0.081 \\
SDI & $1.27(1.1-1.48)$ & 0.001 \\
CCI & $1.09(0.99-1.19)$ & 0.066 \\
Hemoglobin & $0.82(0.72-0.93)$ & 0.002 \\
Creatinine & $1.11(0.99-1.26)$ & 0.073 \\
Albumin & $0.66(0.47-0.91)$ & 0.012 \\
\hline
\end{tabular}

* Government medical insurance. SLE: systemic lupus erythematosus; CSMC: Cedars-Sinai Medical Center; SDI: Systemic Lupus International Collaborating Clinics/American College of Rheumatology Damage Index; CCI: Charlson Comorbidity Index.

be readmitted) but at the expense of not predicting all readmissions.

\section{DISCUSSION}

We have described in detail the clinical and demographic characteristics of 570 inpatient hospitalizations from 455 patients with SLE (of whom 154 were readmitted within 30 days of an index admission) during the course of 2.5 years. As predicted, the risk factors for early readmission included abnormal severity markers of certain laboratory values, mainly low hemoglobin, elevated creatinine, and low albumin, as well as an elevated SDI score. Age and sex did not play a significant role; however, ethnicity significantly differed between the readmitted group and the comparison group. We observed that there was a greater percentage of African Americans and Asians who underwent early readmission. This finding agrees with previous studies in underserved populations, including African American patients, that even after adjusting for a variety of factors, these groups are at highest risk for early readmission ${ }^{12}$. This is of particular importance in SLE because disease manifestations in certain minority populations tend to be more severe, particularly in African Americans, Hispanics, and Native Americans ${ }^{13}$. Also similar to findings in previous publications, patients in the early readmission group, although not statistically significant, were more likely to have Medicaid insurance (OR 1.93, 95\% CI 0.98-3.78). Additionally, we observed that aspects of more severe manifestations of SLE (such as increased SDI, lower hemoglobin, higher serum creatinine, and lower serum albumin levels) representing

Personal non-commercial use only. The Journal of Rheumatology Copyright (C) 2018. All rights reserved. 
significant organ involvement were associated with early readmission, as described by other investigators ${ }^{12}$. Having publicly funded insurance was also a risk factor, suggesting that access to care may play a role. Although the numerical change in albumin levels in both groups appears minute, it was found to be statistically significant. Additionally, a lack of immunosuppressive treatment was protective. The use of these agents most likely indicates that there may be underlying factors of disease severity which we did not measure; the use also indicates the presence of direct effects of these agents suppressing the immune system, leading to an infection risk of early readmission. Patients with SLE who clinically fulfill these associations most likely would warrant more careful attention, especially when considering specialty care followup.

Historically, the complexity and heterogeneity of SLE have posed major challenges in measuring quality, given the diverse features of symptoms and the substantial morbidities that can be seen in these patients. A published study examining administrative data from all inpatient discharges from nonfederal acute care facilities nationwide revealed that $16.5 \%$ of patients with SLE were readmitted within 30 days ${ }^{12}$. Our numbers are almost double this nationwide figure. Because CSMC is a teaching and research SLE center, the higher readmission rate at CSMC could be reflective of a more complex SLE patient population, or a readmission threshold unique to this institution or its location. Previous large studies of SLE hospitalizations using claims databases have provided a wealth of information given their scope and size; however, they are not easily capable of fully examining systematic differences that exist in patients who required early readmission. Moreover, claims databases may lack granular clinical data such as the ability to calculate damage indices, evaluation of medication doses and laboratory values as inpatient and outpatient, and insight into which hospitalizations are truly elective versus unplanned.

Our data permitted substantial insight into the inpatient care of patients with SLE at CSMC. Patient- and system-related factors can be identified with an eye toward a possible prevention strategy to improve discharge plans and timely outpatient services. Key variables that were statistically significant can be used to create a model that functions within an electronic medical record system to identify patients who are at high risk for early readmission. This model would improve transitions in care for high-risk patients and potentially reduce readmission rates in patients with SLE.

There are several strengths to our study. To our knowledge, this is the first study to examine the granular data of patients with SLE who required early readmission. Because we collected detailed clinical information for each individual patient, this enabled risk adjustment for potential confounders. Also, because we used an a priori conceptual framework before data were collected and analyzed, our study is hypothesis testing as well as hypothesis generating.
One limitation is that the study was completely done at CSMC, so it is uncertain whether our study can be generalized to other populations. Additionally, it is possible that readmissions were not recorded if patients presented to other hospitals. Finally, because this is a retrospective study, we may therefore be limited by inconsistencies or even inaccuracies in the way medical information has been recorded. SLE disease duration could not be collected because of these inconsistencies in the medical records.

We are optimistic that the quality of SLE care can be improved. A previously published qualitative study in the SLE underserved population revealed that community-based interventions that improved SLE education as well as facilitated healthcare delivery and psychosocial support were the most likely to improve SLE care ${ }^{14}$.

Using information gathered in our study, we have identified both demographic and clinical risk factors for readmission among patients with SLE. Future directions include development of a model that identifies those patients with SLE at highest risk for hospital readmission prior to the patients' discharge, thereby informing the healthcare team to proceed with mitigation strategies to avoid an unnecessary and costly readmission to the hospital.

\section{REFERENCES}

1. Chakravarty EF, Bush TM, Manzi S, Clarke AE, Ward MM. Prevalence of adult systemic lupus erythematosus in California and Pennsylvania in 2000: estimates obtained using hospitalization data. Arthritis Rheum 2007;56:2092-4.

2. Thorburn CM, Ward MM. Hospitalizations for coronary artery disease among patients with systemic lupus erythematosus. Arthritis Rheum 2003;48:2519-23.

3. Edwards CJ, Lian TY, Badsha H, Teh CL, Arden N, Chng HH. Hospitalization of individuals with systemic lupus erythematosus: characteristics and predictors of outcome. Lupus 2003;12:672-6.

4. Sutcliffe N, Clarke AE, Taylor R, Frost C, Isenberg DA. Total costs and predictors of costs in patients with systemic lupus erythematosus. Rheumatology 2001;40:37-47.

5. Elixhauser A, Steiner C. Readmissions to U.S. hospitals by diagnosis, 2010: statistical brief \#153. Healthcare Cost and Utilization Project (HCUP) statistical briefs. Rockville, MD: Agency for Health Care Policy and Research (US); 2006.

6. Ward MM. Avoidable hospitalizations in patients with systemic lupus erythematosus. Arthritis Rheum 2008;59:162-8.

7. Ward MM. Hospital experience and mortality in patients with systemic lupus erythematosus: which patients benefit most from treatment at highly experienced hospitals? J Rheumatol 2002;29:1198-206.

8. Krishnan E. Hospitalization and mortality of patients with systemic lupus erythematosus. J Rheumatol 2006;33:1770-4.

9. Kossovsky MP, Perneger TV, Sarasin FP, Bolla F, Borst F, Gaspoz JM. Comparison between planned and unplanned readmissions to a department of internal medicine. J Clin Epidemiol 1999;52:151-6.

10. Klabunde CN, Potosky AL, Legler JM, Warren JL. Development of a comorbidity index using physician claims data. J Clin Epidemiol 2000;53:1258-67.

11. Lam GK, Petri M. Assessment of systemic lupus erythematosus. Clin Exp Rheumatol 2005;23(5 Suppl 39):S120-32.

12. Yazdany J, Marafino BJ, Dean ML, Bardach NS, Duseja R, Ward MM, et al. Thirty-day hospital readmissions in systemic lupus

Personal non-commercial use only. The Journal of Rheumatology Copyright @ 2018. All rights reserved. 
erythematosus: predictors and hospital- and state-level variation. Arthritis Rheumatol 2014;66:2828-36.

13. Gomez-Puerta JA, Barbhaiya M, Guan H, Feldman CH, Alarcon GS, Costenbader KH. Racial/ethnic variation in all-cause mortality among United States Medicaid recipients with systemic lupus erythematosus: a Hispanic and Asian paradox. Arthritis Rheumatol 2015;67:752-60.
14. Feldman CH, Bermas BL, Zibit M, Fraser P, Todd DJ, Fortin PR, et al. Designing an intervention for women with systemic lupus erythematosus from medically underserved areas to improve care: a qualitative study. Lupus 2013;22:52-62.

APPENDIX 1. Conceptual model.

\begin{tabular}{lll}
\hline Variable Grouping & Theoretical Variable & Measured Variable in Database \\
\hline Demographic variables & Age & Age in yrs \\
& Comorbidities & CCI \\
& Medication use & Immunosuppressant use \\
& & Steroid use \\
& Sex & Antimalarial use \\
& & Female vs male \\
& & African American \\
& & Asian/Pacific Islander \\
& Insurance & Native American or other \\
& & White \\
& & Medicare \\
Hospitalization characteristics & Type of admission & Medicaid \\
& No. medical procedures & Private \\
& Elective vs nonelective \\
Sisease characteristics & No. inpatient procedures during \\
& & index hospitalization \\
& Organ threatening vs non-organ \\
& Chronic damage & threatening \\
& Presence of SLE-related comorbidities & SDI \\
& & Cardiovascular disease, diabetes, \\
& and malignancy \\
& & Hemoglobin \\
& & Elevated BUN/creatinine \\
& & Low albumin \\
\hline
\end{tabular}

CCI: Charlson Comorbidity Index; SDI: Systemic Lupus International Collaborating Clinics/American College of Rheumatology Damage Index; SLE: systemic lupus erythematosus; BUN: blood urea nitrogen. 